\title{
Opportunistic Information Fusion: A New Paradigm for Next Generation Networked Sensing Systems
}

\author{
Subhash Challa, Tauseef Gulrez, Zenon Chaczko, T.N. Paranesha \\ Information and Communications Group \\ Faculty Of Engineering \\ University of Technology Sydney, Australia. \\ \{schalla, tgulrez, zenon\}@eng.uts.edu.au
}

\begin{abstract}
Traditionally, Information Fusion systems assume that the information is gathered from known sensors over proprietary communication networks and fuse using fixed rules of information fusion and designated computing and communication resources. Emerging technologies like wireless sensor networks, TEDS enabled legacy sensors, ubiquitous computing devices and all IP next generation networks are challenging the rationale of conventional information fusion systems. The technology has matured to a point where it is reasonable to discover sensors based on the context, establish relevance, query for appropriate data, and fuse it using the most appropriate fusion rule, using ubiquitous computing and communication environment in an opportunistic manner. We define such fusion systems as opportunistic information fusion systems. In this paper we introduce this new paradigm for information fusion and identify plausible approaches and challenges to design, develop and deploy the proposed next generation opportunistic information fusion systems.
\end{abstract}

\section{Introduction}

Multi-sensor data/information fusion is a rapidly maturing technology, concerning the problem of combining data/information from multiple sensors to serve different applications. It is a core component of all networked sensing systems, which is used either to:

- Join/combine complementary information produced by sensors to obtain a more complete picture or

- Reduce/manage uncertainty by using sensor information from multiple sources.

Several end applications including environment monitoring, automatic target detection and tracking, battlefield surveillance, remote sensing, etc. depend critically upon data/information fusion [37]. Traditional data/information fusion solutions are designed to work with dedicated sensor and information sources.
Emerging technologies like wireless sensor networks [1], standards enabled legacy sensors

[20,21], ubiquitous computing and communication systems [2,3] and all internet protocol (IP) next generation networks [4] are leading to a new paradigm where sensors can be treated as a shared infrastructure and a common resource with an ability to serve multiple simultaneous data/information fusion applications concurrently. This is in stark contrast to the dedicated sensor, computing and communicating infrastructure serving a specific data/information fusion application. There has been significant progress in dealing with shared computing and communication resources [19]. However, little has been done in regards to treating sensor resources also as a shared resource. Thus, the traditional data/information fusion models, methods, middleware and techniques need to be enhanced/modified to work with sensors that are part of a shared infrastructure. In this paper, we introduce a new paradigm of data/information fusion - the opportunistic information fusion (OIF) paradigm - to handle such multi-purpose sensor networks and services. In addition, we identify several challenges and propose innovations to realize such next generation information fusion systems.

The paper is organized as follows after introduction in section 1, section 2 proposes a new OIF model derived from the well established JDL Model. Section 3, reviews the recently proposed IEEE P1451/TEDS sensor standards and proposes enhancements to support the OIF services. Section 4 proposes new methods of fusing information modeled using heterogeneous uncertainty measures and section 5 proposes opportunistic middleware solutions that can enable the development and deployment of secure, context aware, scalable, flexible and heterogeneous OIF applications. Section 6 illustrates the potential of OIF paradigm using a couple of practical applications. Finally, section 7 concludes the paper and its contributions. 


\section{The New OIF Model}

In the traditional data/information fusion Joint Director of Laboratories (JDL) model is the most popular model in use - especially in the defense applications $[5,6]$. The model breaks down the data fusion processes into five levels ranging from (0-4) consisting of sensor specific signal processing, object refinement and tracking, situation, threat and impact assessment augmented with appropriate databases and fusion rules. One of the fundamental, implicit, assumptions of the JDL model is that the sensors \& the corresponding sensor models are known a priori and are available to serve a particular application in a dedicated manner. Due to this, the data fusion systems, once developed, cannot be re-deployed to serve in contexts other than what it is built for. Thus, when sensors, computing and communications become shared infrastructure and there is no dedicated data fusion application in place, this model is no longer adequate.

\begin{tabular}{|l|l|}
\hline $\begin{array}{l}\text { Conventional } \\
\text { Information Fusion }\end{array}$ & $\begin{array}{l}\text { Opportunistic } \\
\text { Information Fusion } \\
\text { (OIF) Model }\end{array}$ \\
\hline Sensors are assumed & Sensor need to be discovered \\
\hline $\begin{array}{l}\text { Apriori info on sensor } \\
\text { model }\end{array}$ & $\begin{array}{l}\text { Sensor model not known } \\
\text { apriori }\end{array}$ \\
\hline Proprietary networks & Next Gen IP Networks \\
\hline Pre-defined Fusion rules & Determine Fusion Rule \\
\hline $\begin{array}{l}\text { Models of Fusion JDL, } \\
\text { OODA }\end{array}$ & $\begin{array}{l}\text { New/Modified Fusion } \\
\text { Models }\end{array}$ \\
\hline Pre-defined Compt. load & Adhoc Computation Load \\
\hline Fixed middleware & Flexible middleware \\
\hline
\end{tabular}

Table -1: Old Paradigm v/s New Paradigm

Hence a new data fusion model - defined as the Opportunistic Information Fusion Model (OIFM) - to deal with the challenges of realizing the fusion systems in this new paradigm is proposed in figure 1 . The key additions to JDL model that leads to an OIF Model are a new process for establishing relevance and enabling sensor discovery at the lowest level and context enabled re-configuration at all levels of the data fusion process. Even the recently proposed enhancements to JDL model [5] does not support these processes. This new OIF model will enable sensors in a shared infrastructure mode to provide opportunistic services to multiple applications at the same time and deliver new, non-zero sum benefits of information fusion.

Sensor Resource discovery can be achieved by methods like [7] and its relevance can be establishment for the application using the sensors self identification capabilities. Until recently self identification was not an in-built feature in sensors. However, recently, a new standard [20,21,22] promoted by the IEEE instrumentation and measurement working group, enables sensors to have self identification capability. These standards are reviewed and enhancements are proposed in the next section.

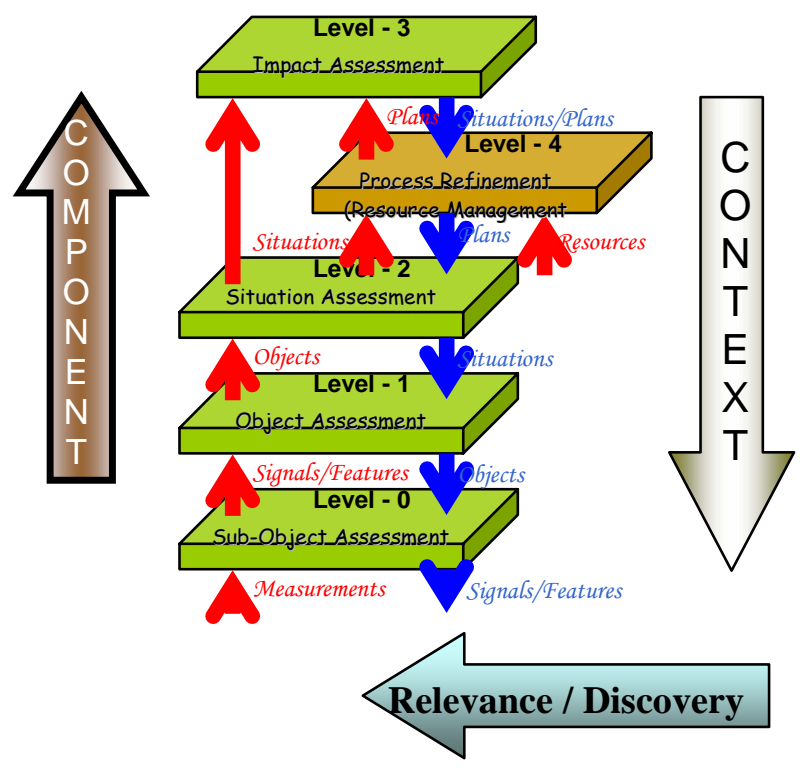

Figure - 1: Proposed OIF Model

\section{IEEE 1451 Standards \& Transducer Electronic Data Sheets (TEDS)}

The OIF model provides a framework for the development of data fusion systems, however, fundamentally, information fusion is carried out mathematically at all levels of information fusion via some kind of uncertain reasoning framework. One of the most successful data fusion techniques is Bayesian data fusion that operates at almost all levels of data fusion. This technique is briefly introduced here and highlights a key requirement of the continued success of this approach in the networked sensing context.

Let ' $t$ ' be the random variable that represents a parameter (e.g., temperature) to be estimated by a sensor network. Let $\left\{Y_{1}, Y_{2}, \ldots, Y_{k}\right\}$ be the set of measurements from sensors $1, \ldots \mathrm{k}$ of any sensor network. The problem 
is to obtain the best estimate of the unknown parameter ' $t$ ', using all the measurements received from the sensors in a sensor network. Within the probabilistic framework the parameter can be estimated from its conditional density using a simple rule:

$\hat{t}=\int t \cdot p\left(t \mid Y_{1}, Y_{2}, \ldots, Y_{k}\right) d t$

where $p\left(t \mid \mathrm{Y}_{1}, \mathrm{Y}_{2}, \ldots, \mathrm{Y}_{\mathrm{k}}\right)$ is the conditional probability density function and the estimate $\hat{t}$ is the best estimate in a minimum variance sense [23]. The Bayesian Fusion rule provides a very elegant way to construct the conditional density from sensor measurements as illustrated below:

$p\left(t \mid Y_{1}, Y_{2}, \ldots, Y_{k}\right)=\frac{1}{\delta} p\left(Y_{1}, Y_{2}, \ldots, Y_{k} \mid t\right) \cdot p(t)$

Assuming $Y_{1}, Y_{2}, \ldots, Y_{k}$ are conditionally independent.

$p\left(t \mid \mathrm{Y}_{1}, \mathrm{Y}_{2}, \ldots ., \mathrm{Y}_{\mathrm{k}}\right)=\frac{1}{\delta}\left\{p_{1}\left(\mathrm{Y}_{1} \mid \mathrm{t}\right) \cdot \mathrm{p}_{2}\left(\mathrm{Y}_{2} \mid \mathrm{t}\right) \cdot \ldots ., \mathrm{p}_{\mathrm{k}}\left(\mathrm{Y}_{\mathrm{k}} \mid t\right)\right\} \cdot p(t)$

where $p(t)$ is the prior distribution of ' $t$ ' assumed by the application. The above formula of multiplying likelihoods $p_{i}\left(\mathrm{Y}_{\mathrm{i}} \mid \mathrm{t}\right)$ of individual sensors with the prior $p(t)$ (and normalised using $\delta$ ) is the so called Bayesian fusion rule [10]. The likelihood encapsulates the error characteristic of the $i$-th sensor. Thus the likelihood function (or some other uncertainty representation function like fuzzy membership function) that captures the sensors' uncertainty characteristic is vital for successful information fusion. Traditional IF systems use dedicated sensors with known a priori likelihood functions. However in OIF systems, as sensors are part of a shared infrastructure and are not known a priori, this information on likelihood functions (uncertainty measures) needs to be gathered directly from sensors (upon discovery).

In a networked sensing context, when sensors come in and go out of service in an ad hoc manner, sensor discovery is not trivial if the sensors do not have self-identification and sensor service registration capability. Transducer Electronic Data Sheets (TEDS) is the heart and soul of a new IEEE 1451 family of smart transducer interface standards which are introduced by IEEE instrumentation and measurement working group for standardization of Smart Transducer Interfaces and a set of common interfaces [20,21]. TEDS contains the critical information needed by an instrument or measurement system to identify, characterize, interface and properly use the signal from the smart sensor. These key features are of great importance in the OIF context as they provide the sensors with self-identification capability on a sensor network. This self-identification capability is critical to opportunistic methods of sensor service discovery.

TEDS utilizes the concept of templates as shown in the Table 2 that defines the specific properties for different sensor types. The Basic TEDS field has all the vital information like Manufacturers ID, sensor type etc that can enable context based sensor discovery. This research proposes to develop new methods of sensor service discovery by using the basic TEDS and user area information in conjunction with the contextual inputs to solve the relevant sensor discovery problem.

From the Bayesian Fusion rule it is evident that the likelihood information needs to be gathered from the sensors directly upon discovery. It is sometimes possible to approximate likelihood functions (uncertainty measures) from the available data from TEDS template using the Standard TEDS information (available in second field) along with the data generated by the sensor to serve the information fusion application. However, such derived functions are, at best, approximations and can lead to erroneous fusion results. As this is true sensor error characteristics and is the proprietary information of sensor manufacturer, it is better if it is a part of the standard and has it supplied directly by sensor suppliers/manufacturers as a part of standard TEDS template. As current approved standards do not incorporate such uncertainty descriptor functions, we propose the following modification to the standard TEDS field (showed in Bold in second field) to enable opportunistic information fusion.

\begin{tabular}{|l|l|l|}
\hline Basic TEDS & Manufacturer ID & 43 \\
& Model ID & 7115 \\
& Version Letter & $\mathrm{B}$ \\
& Serial Number & $0073 \mathrm{IF}$ \\
\hline Standard & Calibration Date & Jan. 29,2000 \\
TEDS & Sensitivity @ & $1.094 \mathrm{E}+03 \mathrm{mV} / \mathrm{g}$ \\
(IEEE & Reference Freq, & $100.0 \mathrm{~Hz}$ \\
Accelerometer & Temp, Meas. Range, & $23^{\circ} \mathrm{C}$ \\
Sub-template) & Electrical Output & $\pm 50 \mathrm{~g}$ \\
& Quality Factor & $\pm 5 \mathrm{~V}$ \\
& Temp Coefficient & $300 \mathrm{E}-3$ \\
& Direction (X, Y, Z) & $-0.48 \% /{ }^{\circ} \mathrm{C}$ \\
& Reasoning Frame & Probability \\
& Likelihood & Normal (0,1) \\
\hline User Area & Function. & \\
\hline & Sensor Location & Building 2-lvl:2 \\
& Calibration Due & April 15, 2002 \\
\hline
\end{tabular}

Table 2 - Proposed TEDS Template 


\section{Opportunistic Information Fusion with Heterogeneous Uncertainty Measures}

The freedom to supply uncertainty measures within the respective TEDS fields opens it up to heterogeneous choice of uncertainty measures. The uncertainty measures can be one of probability, possibility (fuzzy) or belief measures that capture and represent the uncertainty in sensor measurements in different approximate reasoning frameworks. In such a scenario it is possible to encounter situations where sensors in the networked data/information fusion application can have heterogeneous uncertainty measures. Seminal works by Oxenham, Challa and Moreland [8] propose distributed information fusion methods that deal with heterogeneous uncertainty measures with built-in uncertainty preserving transformations to move from one uncertainty reasoning framework to the other without changing the level of uncertainty. For example the pignistic and inverse pignistic transformations proposed by Phillipe Smets $[12,13]$ have been used in [8] to move between probability and belief measures before fusion as elucidated below.

The Pignistic probability BetProb is calculated from a belief function $\left(F^{\prime}, m\right)$ by setting $\operatorname{BetProb}\left(x_{j}\right)=$ $\sum_{F \in F^{\prime} \mid x_{j} \in F} m\left(F^{\prime}\right) / F^{\prime} \mid(1-m(\phi))$ for each $x_{j}$ in the frame of discernment $\Omega$ [36, p. 202]. The Inverse Pignistic belief mass can be calculated by first letting the elements $x_{j}$ of $\Omega$ be re-labelled such that:

$p\left(x_{j 1}\right) \geq p\left(x_{j 2}\right) \geq \ldots \geq p\left(x_{j k}\right)$, where $k=|\Omega|$ for each $r=1, \ldots, k$ define the subset:

$\hat{F}_{r}=\left\{x_{j 1}, x_{j 2}, \ldots, x_{j r}\right\}$, and assign it the mass:

$\hat{m}\left(\hat{F}_{r}\right)=\left|\hat{F}_{r}\right| \cdot\left(p\left(x_{j r}\right)-p\left(x_{j r+1}\right)\right), \quad$ where $p\left(x_{j k+1}\right)=0$ by convention. Then the focal elements of $\left(\hat{F}^{\prime}, \hat{m}\right)$ are the (nested) sets $\hat{F}_{r}$ which have a non-zero mass $\hat{m}\left(\hat{F}_{r}\right)$ assigned to them. This work is, however, limited to Bayesian and Dempster-Shafer reasoning frameworks. To realize the full potential of the OIF paradigm, these ideas need to be extended to fuzzy/possibility theory frameworks as well.

One of the approaches we suggest in handling such heterogeneous uncertainty measures, including fuzzy measures, is to consider Random
Sets framework. In the late 1990s, Ron Mahler proposed random sets as a means of probabilistically modeling versions of crisp and fuzzy Dampster-Shafer theory in a way that is consistent with the Bayes' fusion rule [17, 18]. There are three steps in such an approach to deal with data that has inherent uncertainties [15,16,17]. First, such evidence/data is modeled as a random closed subset $\Theta$ of the underlying measurement space. Second, some modelling technique-fuzzy logic, Dempster-Shafer theory, rules-is used to construct $\Theta$. Third, a generalized likelihood function $\rho(\Theta \mid \mathbf{x})$, based on an ambiguous signature model base and a data-to-model matching technique, is used to hedge against uncertainties both in data-modeling and in the modeling of data generation. The generalized likelihood function is then used in the Bayesian Fusion rule - like the one introduced in equation (1).

Here we demonstrate modeling fuzzy membership function using random set. To construct useful models of $\Theta$ one could use a fuzzy-set modeling process, in which ambiguity in the data $\mathbf{z}$ is modeled as a fuzzy membership function $g(\mathbf{z})$ on measurement space. Let $A$ be a uniformly distributed random number in $[0,1]$. Then the random subset

$$
\Theta=\Sigma_{\mathrm{A}}(\mathrm{g})=\{\mathbf{z} \mid \mathrm{A} \leq \mathrm{g}(\mathbf{z})\}
$$

contains the same information as the fuzzy model $g(\mathbf{z})$. A similar approach has been shown to convert a basic belief mass assignment to a random closed set in $[15,16,17]$. However, such modeling has not yet been used to fuse information from multiple sensors where each sensor represents information in a different uncertainty measure.

We propose to use a basic methodology that will first convert the non-probabilistic uncertainty measure, like fuzzy membership function or basic belief mass assignment, into a closed random subset and instantiate the Bayesian Fusion approach to combine the data. One of the key questions that has not yet been answered in literature and which has direct implication on the use of these techniques of information fusion is the relationship between level of uncertainty in the closed random set representation and the level of uncertainty in the fuzzy membership or basic belief mass assignment.

\section{Middleware for Opportunistic Information Fusion}

The term Middleware relates to software system infrastructure that constitutes a set of services that aim at facilitating the development of distributed applications 
in heterogeneous environments. Middleware is a software layer that is placed above the operating system - including the basic communication protocols - and below the distribution applications that interact via the network. Its primary objectives are to foster portability and interoperability of distributed application components. The middleware layer allows software components to exchange data and interact with one another regardless of the underlying communication protocols, operating systems and hardware platforms on which the components reside. This is made possible through the use of standard application programming interfaces (APIs) and services.

Mainstream middleware solutions traditionally offer a useful, distributed programming models and solutions that mask the heterogeneity of networks, end-systems, operating systems, programming languages and hardware. Middleware solutions such as CORBA, RMI, J2EE, .Net, etc. have been very successful in business applications; wrapping of legacy systems and many other tasks [24, 26, 28]. However, none of these can adequately address the requirements posed by the new opportunistic information fusion paradigm such as: serendipitous sensor service discovery, situation and context awareness, opportunistic information access and delivery, contextual interpretation and presentation, support for heterogeneous capabilities of individual network devices, and reliable support for privacy, rights, security and trust. Hence a new Opportunistic Middleware model (OMM) is proposed.

The Figure- 2 depicts the essence of the proposed opportunistic services in OMM. To obtain an opportunistic SN service the user issues a query which is analyzed in the Context Engine. If a query request is contextually feasible then the request to discover sensor(s) is issued to the Opportunistic Data Fusion Framework. Next the process involves establishing relevance, feasibility and quality of service delivery. Here pivotal role in establishing service relevance is played by information contained in TEDS. The Relevance Correlation process aims at finding compatibility between information Fusion Rules, discovered (accessible) sensors and the TEDS fields which contain sensors' physical and logical parameters. Upon finding opportunistic sensor service and establishing relevance, the service instance is registered as a Sensor Service Registry.

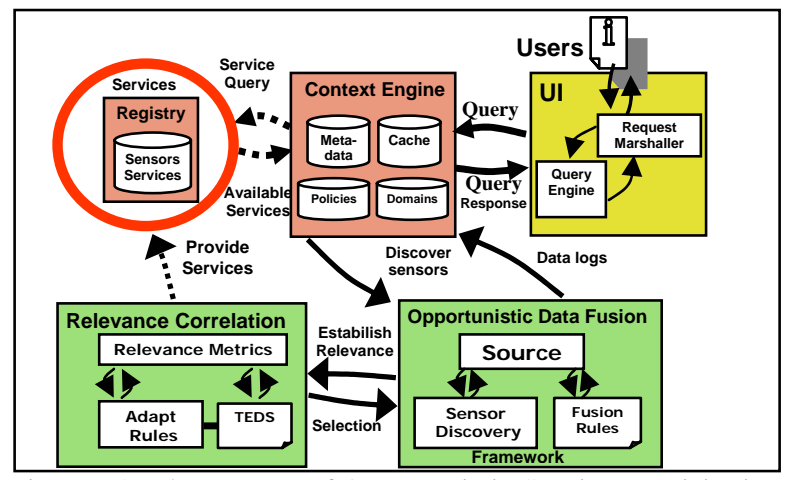

Figure - 2: The process of Opportunistic Service Provisioning

\subsection{The Opportunistic Middleware Model}

The OMM is to support Sensor Network (SN) solutions that are autonomic in nature, scalable, and flexible. The model should support autonomic computing $[25,27]$ that is characterized by a set of "self-x" properties such as: self-organizing, self-configuring, self-healing, self-protecting and self-optimizing along with aspects of context and situation awareness.

The proposed OMM aims to deliver software system framework that, apart from traditional middleware goals, attempts to achieve the following:

- Provide Support for Opportunistic Sensor Discovery

Opportunistic sensor service discovery aims to gather information wherever and whenever it is useful and relevant. Some sensors can provide duplicate information while others can be found by a chance. The proposed OMM needs to deal with aspects of sensors data redundancy and serendipity.

- Provide Support for Opportunistic Data Access.

Users should be able to interact with information/other users with ease and in various modes (i.e. fast data paths, slow data paths). High performance hardware should be supported to provide opportunistic services at Sensor Network Access Points.

\section{- Provide Support for Opportunistic Interpretation.}

Computer resources and computational tools should be used to make information and interactions meaningful to end users and to provide mechanisms for context interpretation and awareness.

In contrast to the classical, adaptive, reflective and ad-hoc middleware models [29, 30, 31] adopted for architectures of most NS-based systems [28] the opportunistic middleware model is to promote notions of 
"negotiable connectivity", "right to reject" connections to undesirable nodes and "compromise on principles" to achieve mission critical goals. In contrast to ad-hoc concepts, the opportunistic middleware model is to support pro-active, goal-oriented behaviour that moderates prohibitive aspects of worst-case scenarios yet respecting individual and varying capabilities of network services, links and devices. Undesirable connections in the sensor based systems may relate to connections which are perceived as irrelevant/"non-contributive" to OIF, those which could potentially drain available resources (i.e. energy, bandwidth or computing power) or those perceived to be disruptive in context of achieving the mission that is being executed by the system.

These selective and adaptive connectivity measures/policies built-in the OMM allow a system to operate in self-defense mode scheme if such a need arise. In pessimistic scenarios, the connectivity policies would test for possible degradation of sensor services upon a new sensor connection (and use the "right to reject” or "compromise on principles" rules) while in optimistic scenarios, the policies could check for possible improvements when a new sensor connection opportunity occurs and "accept" the new element into its definition of "service". Alternatively, consensus policies (the "negotiable connectivity" rule), might be applied where scenario dichotomies are weighted for possible overall improvement or degradation in sensor services. OMM needs to be built to:

- Handle unpredictable dynamic changes as they occur (i.e. addition/ deletion of devices and services)

- Deal with various resources as they are discovered facilitate inter-device cooperation between devices which do not know about each other

- Provide connectivity for devices that are available intermittently

- Support heterogeneous devices/ networks and services that are deployable in a simple, flexible and secure manner

- Support for context awareness. provide mechanisms that ensure maximum survivability, reduction of environmental load by information devices and

- Support bandwidth and energy optimizations

- Should involve opportunistic compensation of resource failures

- Provide opportunistic reconfiguration of computation resources at the level of remote access points, sensors and actuators

- Support self-organization (i.e. ad hoc network formation, autonomous and serendipitous connection establishment, etc.).

While catering for the above requirements, the proposed OMM needs to cope with performance issues arising due to:

- The number of sensors and users may fluctuate and be unpredictable.

- If users cannot get responses in real time, they may abort requests.

- The sensors forming a SN may belong to independent autonomous organizations that do not necessarily trust each other.

- Further, interaction takes place on an insecure medium.

- The communication infrastructure (all IP back bone network) does not provide QoS guarantees.

- Because of the inherently open environment, retrieving sensor information can demand self-describing data (TEDS) and agreement on common ontology.

- New SN applications must interoperate seamlessly with existing legacy applications.

\subsection{Open Challenges in OMM}

There are still many open questions and issues which need to be addressed when implementing the proposed OMM. For example context-aware applications need selective transparency features. Complete distribution transparency is inappropriate when SN applications must adapt to fluctuations in resource supply such as variations in communication bandwidth and fading battery power. However, we do not currently have middleware facilities to control the degree of transparency. One of the ways to achieve this is for middleware systems to renounce the strict OSI layering principle and support direct interaction between nonadjacent layers. In addition OMM must support dynamic changes in system configuration and operating context at runtime. OMM must monitor the computing and communication resource supply and demand, compute adaptation decisions and notify applications if they require adaptation.

Existing middleware platforms do not scale to the device diversity, size and runtime dynamics that OIF applications demand. Middleware research and development is currently undergoing a transition addressing several important questions like: 
- What is the most appropriate programming model? Is a single distributed programming model sufficient?

- Can we build customizable, configurable and flexible middleware frameworks for inherently heterogeneous OIF paradigm?

- What are the desirable middleware features and infrastructure

\subsection{Privacy, Rights, Security and Trust}

Privacy, Rights, Security and Trust (PRST) [32, $33,34]$ are integral parts of OMM that address important issues in opportunistic SN systems. The opportunistic middleware needs to develop original strategies and policies based on such probabilistic PRST models. The real challenges in fact lies in finding useful PRST models which could scale up, support autonomous decision making and be flexible [31]. The popular deterministic PRST models may need to give way to probabilistic security models.

\section{Emerging Applications of OIF}

A couple of example applications where OIF can add significant value are presented below.

\subsection{Homeland Security}

When tracking suspected criminals or law breakers the law enforcement would like to deploy adequate resources to monitor, identify, capture and detain the subjects. However, most of the current solutions do not consider bring the already deployed sensors to service the task. Consider a scenario where a subject is tracked well it hits a shopping complex. It is not unusual that in such built environments it is much easier to hide and avoid capture. However, if the already installed sensors are assumed to TEDS enabled and are part of an accessible network (most of the installed cameras are already on a network), it is very much possible to query these sensors in an opportunistic manner to obtain data of relevance to meet the objectives of capturing the subject.

\subsection{Robot Obstacle Avoidance}

The robotic obstacle avoidance is a critical portion of robotic path-planning. The basic concept is to use the opportunistic information available from already installed sensors in the environment in conjunction with the onboard sensor information to addressing the obstacle avoidance problem. As shown in figure - $3 \& 4$, robot seeks the depth of obstacle. As its onboard sensors are insufficient to provide it, the robot pings to the next generation, all IP back bone network to obtain a complementary view from the already installed security camera, which resolves its problem. The fused information is appropriate in providing a more accurate and elegant solution to the path-panning problem.

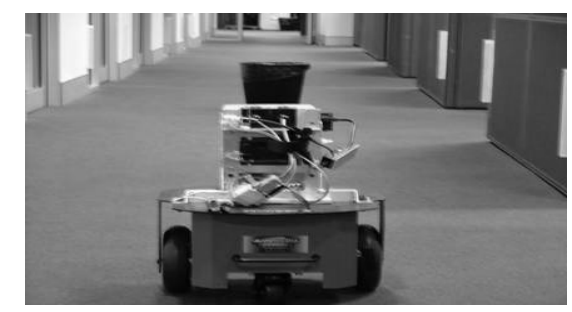

Figure - 3: Robot encounters an obstacle in front.

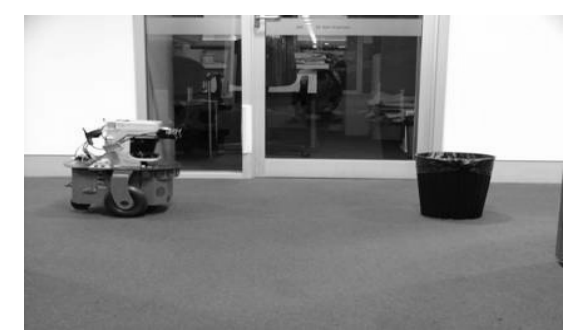

Figure - 4: Robot pings to the off-board relevant camera to get the depth of the obstacle.

\section{Conclusions}

In this paper we have introduced a new paradigm of information for the design and development of next generation data fusion systems. New data fusion models are and modifications to emerging TEDS standards are proposed. The issues and challenges associated with fusion of heterogeneous uncertainty measures and Middleware design have been identified. Context representation, awareness and their use in the reconfigurable middleware is highlighted. The potential for the new OIF paradigm is then illustrated in the context of a few applications.

\section{References}

1. Smart sensors in silicon : URL: http://www.xbow.com/

2. T. Nakagawa, T. Yoshikawa, K. Ohta, H. Inamura and S. Kurakake, (ASWN2004) Design and Evaluation of Scalable Ubiquitous Discovery System NTT DoCoMo, Inc., Japan.

3. U. Varshney and R. Jain,"Issues in Emerging 4G Wireless Networks. “ MUM 2002 - 1st International Conference on Mobile and Ubiquitous Multimedia.

4. IPv6 and Hitachi, http://www.internetworking.hitachi.com

5. James Llinas, Christopher Bowman, Galina Rogova , Alan Steinberg, Ed Waltz and Frank White "Revisiting 
the JDL Data Fusion Model II”. In Proccedings of The 7th International Conference on Information Fusion, 2004 Stockholm, Sweden

6. F. White, "A Model for Data Fusion", Proc. of the 1st Int. Symposium on Sensor Fusion, 1988.

7. Christian Frank, Vlado Handziski, Holger Karl. "Service Discovery in wireless sensor networks". Technical report TKN-04-006, Telecom. Group,Technical Univ. Berlin, March 2004.

8. Martin G. Oxenham, Subhash Challa \& Mark Morelande, “Fusion of Disparate Identity Estimates for Shared Situation Awareness in a Network-Centric Environment". Submitted to: $8^{\text {th }}$ Information Fusion Conference July 2005.

9. S. Challa and D.Koks, "Bayesian and Dempster-Shafer fusion" Sadhana Vol. 29, Part 2, April 2004, pp. 145-174.

10. S. Challa, B.N. Vo and X. Wang, "Bayesian approaches to track existence - IPDA and random sets. In Proc. $5^{\text {th }}$ Int'l conf. on Information Fusion, San Diego, June 2001. ”

11. K. Hestir, H.Nguyen and G.Rogers, "A random set formalism for evidential reasoning". In I. Goodman, M. Gupta, H. Nguyen and G. Rogers, eds., Conditional Logic in Expert Systems, 309-344, North-Holland, Amsterdam, 1991.

12. Phillippe Smets. "Constructing the pignistic probability function in a context of uncertainty." $5^{\text {th }}$ annual conf. on Uncertainty in Artificial Intelligence, NY 1990.

13. Philippe Smets. "The necessity of the pignistic transformation". Preprint 2004.

14. R. Mahler. "Combining Ambiguous Evidence With Respect to Ambiguous a priori Knowledge, II: Fuzzy Logic”. Fuzzy Sets and Systems, 75: 319-354, 1995.

15. R. Mahler. "Combining Ambiguous Evidence With Respect to Ambiguous a priori Knowledge, I: Boolean Logic”. IEEE Trans. SMC-Part A, 26: 27-41, 1996.

16. R. Mahler. "Representing rules as random sets, I: Statistical correlations between rules". Information Sciences, 88: 47-68, 1996.

17. R. Mahler. "Random Sets: Unification and Computation for Information Fusion - A Retrospective Assessment" $8^{\text {th }}$ Conf. on Inf. Fusion, Stockholm, 2004.

18. D. Fixsen and R. Mahler. "The modified Dempster-Shafer approach to classification”. IEEE Trans. SMC-Part A, 27(1): 96-104, 1997.

19. Xin Liu, Edwin K. P. Chong, and Ness B. Shroff, "Opportunistic Transmission Scheduling With Resource-Sharing Constraints in Wireless Networks". IEEE Journal on selected areas in communications, vol. 19, NO. 10, OCTOBER 2001

20. Kang Lee. "IEEE 1451: A Standard in Support of Smart Transducer networking". National Institute of Standards and Technology 100 Bureau Drive Stop 8220 Gaithersburg, Maryland 20899-8220 USA.

21. "IEEE Std 1451.2-1997, Standard for a Smart Transducer Interface for Sensors and Actuators -
Transducer to Micmprocessor Communication Protocols and Transducer Electronic Data Sheet (TEDS) Formats," Institute of Electrical and Electronics Engineers, Inc., Piscataway, New Jersey 08855, September 26, 1997.

22. "IEEE Std 1451.1-1999, Standard for a Smart Transducer Interface for Sensors and Actuators - Network Capable Application Processor (NCAP) Information Model," Institute of Electrical and Electronics Engineers, Inc., Piscataway, New Jersey 08855, June 25, 1999.

23. A. Jazwinski, "Stochastic Processes and Filtering Theory”, Academic Press, New York, 1970.

24. Campbell A., G. Coulson G., Kounavis M., "Managing Complexity: Middleware Explained." IT Professional, IEEE Computer Society, 1:5, September/October 1999, 22-28.

25. Bantz D. F. et al, Autonomic personal computing, Autonomic Computing, Volume 42, Number 1, 2003.

26. Bernstein P., "Middleware: A Model for Distributed System Services", Communications of the ACM, 39:2, February 1996, 86-98.

27. Ganek, A and Corbi, T. "The Dawning of the Autonomic Comuting Era”. IBM Systems Journal, 42(1), 5-18.

28. Hill J., Szewczyk R., Woo A., Hollar S., Culler D., and Pister K., "System Architecture Directions for Networked Sensors". In ASPLOS 2000, Cambridge, USA, Nov. 2000.

29. Mahmoud Q. H., "Middleware for Communications", West Sussex, Wiley \& Sons, 2004.

30. Schantz, R.E. and Schidt, D.C. "Middleware for Distributed System: Evolving The Common Structure for Network-centric Applications”, Ecyclopedia of Software Engineering, New York, Wiley \& Sons, 2001, 801-813.

31. Geihs, K., "Middleware Challenges Ahead", IEEE Computer, vol 36(0), pages 24-31.

32. Roy Campbell, Jalal Al-Muhtadi, Prasad Naldurg, Geetanjali Sampemane, M. Dennis Mickunas, "Towards Security and Privacy for Pervasive Computing”, Theories and Systems, Mext-NSF-JSPS International Sympsoium, ISSS 2002, Tokyo, Japan.

33. V. Cahill, B. Shand, E. Gray, N. Dimmock, A. Twigg, J. Bacon, C. English, W. Wagealla, S. Terzis, P. A. Nixon, C. Bryce, G. Serugendo, J. Seigneur, M. Carbone, K. Krukow, C. Jensen, Y. Chen, and M. Nielsen, "Using Trust for Secure Collaboration in Uncertain Environments", IEEE Pervasive Computing Magazine, 2003.

34. M. Langheinrich, "A Privacy Awareness System for Ubiquitous Computing Environments", in UBICOMP 2002, LNCS 2498, pp 237-245, 2002.

35. Waleed Wagealla, Sotirios Terzis and Colin English, "Trust-based Model for Privacy Control in Context-aware Systems", in UBICOM Workshop on Security in Ubiquitous Computing, 2003.

36. Philippe Smets and Robert Kennes. "The transferable belief model”. Artificial Intelligence, 66:191-234, 1994.

37. Anna Hac. "Wireless Sensor Network Designs", John Wiley and Sons, Ltd, ISBN 0-470-86736-1. Chapter 2, pg no 31-32. 